\title{
Programa de formación resiliente en hábitos y actitudes hacia el estudio en estudiantes universitarios
}

Resilient training program in habits and attitudes towards study in university students

Programa de treinamento resiliente em hábitos e atitudes em relação

ao estudo em estudantes universitários

\author{
FREDDY BARDALES URIARTE
}

\begin{abstract}
RESUMEN
Esta investigación tuvo como objetivo general determinar la influencia del programa de formación resiliente en hábitos y actitudes hacia el estudio en alumnos universitarios de Piura, 2018. El diseño de investigación fue cuasi experimental debido a que los grupos ya estaban definidos. La población estuvo conformada por 290 estudiantes de la Escuela de Psicología de una universidad particular de Piura. La muestra estuvo constituida por 70 estudiantes universitarios. El muestreo utilizado fue no probabilístico por conveniencia; debido a que el acceso a los evaluados estuvo sujeto a su disponibilidad y viabilidad según sus horarios. La técnica del instrumento utilizado fue la encuesta. Como instrumento se utilizó el cuestionario ENCUESTA DE HÁBITOS Y ACTITUDES HACIA EL ESTUDIO (EHAHE) adaptado a la población de México por Fernando García Cortes y Eduardo García Hassey. Está dividido en siete subescalas, consta de 100 ítems. Se aplicaron 12 sesiones de trabajo a manera de taller para la formación resiliente en los estudiantes. Finalmente, concluye que el programa de formación resiliente influye en los hábitos y actitudes hacia el estudio en los estudiantes universitarios de Piura.
\end{abstract}

Palabras clave: Resiliencia; educación; enseñanza y formación; método de aprendizaje.

\begin{abstract}
This research had the general objective of determining the influence of the resilient training program on habits and attitudes towards study in university students from Piura, 2018. The research design was quasi-experimental because the groups were already defined. The population was made up of 290 students from the School of Psychology of a private university in Piura. The sample consisted of 70 university students. The sampling used was non-probabilistic for convenience; because access to the evaluated was subject to their availability and viability according to their schedules. The technique of the instrument used was the survey. The HABITS AND ATTITUDES TO THE STUDY SURVEY (HATSS) adapted to the population of Mexico by Fernando García Cortes and Eduardo García Hassey was used as an instrument. It is divided into seven subscales, consisting of 100 items. Twelve work
\end{abstract}


sessions were applied as a workshop for resilient training in students. Finally, it is concluded that the resilient training program influences the habits and attitudes towards study in university students in Piura.

Keywords: Resilience; education; teaching and training; learning method.

\section{RESUMO}

Esta pesquisa teve como objetivo geral determinar a influência do programa de treinamento resiliente nos hábitos e atitudes em relação ao estudo em estudantes universitários de Piura, 2018. O desenho da pesquisa foi quase experimental, porque os grupos já estavam definidos. A população era composta por 290 estudantes da Faculdade de Psicologia de uma universidade particular de Piura. A amostra foi composta por 70 estudantes universitários. A amostragem utilizada não foi probabilística por conveniência; porque o acesso aos avaliados estava sujeito à sua disponibilidade e viabilidade de acordo com seus horários. A técnica do instrumento utilizado foi a pesquisa. Os HÁBITOS E ATITUDES PARA A PESQUISA DE ESTUDO (HAPPE) adaptados à população do México por Fernando García Cortes e Eduardo García Hassey foram utilizados como instrumento. É dividido em sete subescalas, compostas por 100 itens. Doze sessões de trabalho foram aplicadas como um workshop para treinamento resiliente em estudantes. Finalmente, conclui-se que o programa de treinamento resiliente influencia os hábitos e atitudes em relação ao estudo em estudantes universitários de Piura.

Palavras-chave: Resiliência; Educação; ensino e treinamento; método de aprendizagem.

\section{INTRODUCCIÓN}

La problemática educativa aqueja y preocupa en muchos lugares del mundo. García \& Rodríguez (2013) refieren en su investigación realizada en la Universidad Distrital Francisco José de Caldas en la ciudad de Bogotá- Colombia, que gran parte de los estudiantes que fueron investigados no tenían claridad respecto al conocimiento del curso (nivel cognoscitivo), de igual forma se denota la falta de interés al estudio por parte del alumnado (nivel afectivo), de la misma manera los estudiantes presentaban una actitud de total resignación por la materia, ya que ninguno mostraba interés en desarrollar las actividades propuestas (nivel conativo). A nivel de comportamiento los estudiantes mostraban actitudes de irrespeto y desobediencia ya que no visualizan una figura de autoridad en sus docentes.

La realidad peruana no es ajena a lo referido anteriormente, puesto que uno de los indicadores que se puede apreciar a nivel nacional en las universidades del Perú es que solo se enfocan en la formación académica a nivel cognitivo, más no a nivel actitudinal lo cual genera un inadecuado comportamiento de algunos estudiantes debido a la presión académica y a la falta de una resiliencia que erradique el estrés y eleve el bajo nivel de práctica de actitudes positivas que tienen los estudiantes, por la falta de un adecuado sistema de evaluación actitudinal y programas de resiliencia que ayuden a erradicar el estrés que trae a los estudiantes las labores académicas y la proximidad de los exámenes.

Por otro lado, se puede mencionar el caso de algunas universidades que se preocupan por 
lograr en los estudiantes algunas mejoras en su formación actitudinal, en aspectos como el orden, puntualidad, deseo de superación y responsabilidad. Entre algunas universidades está la Universidad Privada del Norte, donde la calificación mínima aprobatoria es 12; mientras que en la Universidad Peruana de Ciencias Aplicadas (UPC) es 13; y en la Universidad Católica Santo Toribio de Mogrovejo de Chiclayo, el calificativo mínimo aprobatorio es 14. Estos dispositivos establecidos en sus reglamentos del Estudiante, conocidos y aceptados por sus alumnos, además de publicados en sus portales de Transparencia, permiten mejorar aspectos actitudinales en concordancia con su desempeño académico. Sin embargo, esto no es suficiente para impulsar la mejora de las actitudes en los estudiantes universitarios.

A nivel local, la resiliencia y afectividad en los estudiantes universitarios de los primeros ciclos está en un nivel bajo de acuerdo con la información recibida por el Director de Escuela Profesional de la institución objeto de estudio, quién manifestó que si bien es cierto que se contempla las evaluaciones actitudinales dentro de los sílabos de los cursos lectivos de los tres primeros ciclos, sin embargo, no se lleva en la práctica tal evaluación actitudinal y según la evaluación de tutoría que reciben en dichos ciclos, ésta solamente se basa en el rendimiento cognitivo y no en lo actitudinal, demandando un bajo nivel de resiliencia. Existe, por lo tanto, un descuido en el trabajo actitudinal que realizan los docentes. De acuerdo con las consideraciones antes expuestas, se identificaron las siguientes dificultades en gran parte de los estudiantes; como son: bajo nivel de afectividad, algunos no respetan las opiniones y decisiones de sus compañeros, muestran timidez cuando se les pide su opinión con respecto a un tema, no valoran los aportes e ideas de los demás, dificultad en expresar sus sentimientos y opiniones ante sus compañeros de forma asertiva, inadecuado uso de la razón para tomar decisiones en las clases, dificultad para afrontar problemas académicos, deficiencia para ser condescendientes con sus compañeros, deficiencia para cumplir con las normas establecidas por los docentes, demuestran comportamientos inadecuados en el aula, inadecuada orientación de sus actitudes ante sus compañeros, dificultad para trabajar en equipo.

Frente a los problemas dentro de la unidad de estudio se encuentran educandos poco comprometidos con la carrera que estudian, inestables emocionalmente, dinámica familiar disfuncional, familias monoparentales, dificultad en la comprensión lectora, poca tolerancia a la frustración, abandono prematuro de su carrera profesional o también conocida como deserción académica universitaria.

Así también se resalta que bajo las dificultades referidas afecta en el comportamiento actitudinal de los estudiantes generando un bajo nivel en el rendimiento académico, afectando su desenvolvimiento y seguridad de sí mismo, no permitiendo su desarrollo académico profesional.

Se deduce que no existe una adecuada eficiencia de los docentes para trabajar el componente actitudinal y que Tutoría, a pesar del trabajo que realizan, no es suficiente para elevar el nivel de resiliencia y mejorar el nivel actitudinal de los estudiantes universitarios de una universidad de la ciudad de Piura. Por todo lo expuesto, urge la implementación de un programa de formación en resiliencia para la mejora en hábitos y actitudes hacia el estudio en educandos de una universidad particular de Piura.

Por otro lado, respecto a los fundamentos teóricos, podemos ver que en referencia a los há- 
bitos de estudio (Escobar, 1996) refiere que influyen métodos y técnicas para sacar el máximo provecho del aprendizaje. Ya que a través de su empleo el alumno ejercita la memoria, retención y aplicación de los contenidos (conceptuales, procedimentales, actitudinales). Así también el autor refiere que el estudiante en los primeros niveles académicos, deberá ser entrenado en el uso y la adquisición de los hábitos de estudio, los cuales le son indispensables en la educación media, posteriormente en niveles superiores y en el ámbito laboral.

Respecto a los indicadores de hábitos de estudio está: La evasión-retraso y Los métodos de trabajo; respecto a lo primero está orientado a la rapidez de una persona para terminar los trabajos escolares, su facilidad para concentrarse y no perder el tiempo en distracciones inútiles. En parte el segundo indicador (Los Métodos de trabajo), está referida al empleo de procedimientos de estudio efectivo; la eficacia para hacer los trabajos escolares. Con respecto a las actitudes hacia el estudio (Corella, 1996) lo define como la respuesta afectiva del alumno hacia los nuevos contenidos escolares, sus maestros y las actividades que le impone la escuela, así como las creencias que éste tiene respecto a las potencialidades de los maestros y de la escuela.

Respecto a los indicadores de actitudes hacia el estudio (Corella, 1996) refiere que son dos indicadores: la aprobación del maestro y la aceptación de la educación. Esta primera está referido a la opinión que los alumnos tienen acerca de los maestros y de las actividades de estos en el salón de clases. Sin embargo el segundo indicador referido a la aceptación de la educación, hace referencia a la aprobación en relación por las metas, prácticas y requerimientos educativos (el alumno estudia por igual todas sus materias independientemente de su relación con el maestro o si le gusta o no el tema, elige los temas y reportes con habilidad para sus trabajos, lee con interés y atención sus libros de texto, piensa que las calificaciones son justas y reflejan la capacidad del alumno, piensa que los estudios le dan elementos para su vida adulta y estudia para aprender).

Por otro lado, lo referente a los hábitos hacia el estudio, son las actuaciones o las constantes, efectivas y eficientes con las que el alumno se relaciona ante nuevos contenidos de aprendizaje, con el propósito de conocerlos y especialmente aplicarlos en su vida cotidiana, para obtener un buen rendimiento escolar, buenas calificaciones, certificado de bachillerato, una beca o un título (Brown, 1973).

Grotberg (1995), respecto a la resiliencia, refiere que es la habilidad del ser humano para afrontar las dificultades de la vida, instruirse de ella, superarlas e incluso ser transformados por las mismas. Todas las personas pueden ser resilientes si así se lo proponen, si realmente desean transformar su vida y tiene ganas de luchar; el desafío está en encontrar la manera de fomentarla, tanto a nivel de uno mismo como familiar o social.

Vanistendael \& Lecomte (2002) mencionan que la resiliencia es como la fortaleza a la destrucción, es decir la habilidad de proteger la propia integridad, bajo presión y por otra parte, la define como la capacidad para crear un comportamiento vital positivo pese a las adversidades. 
Por otro lado, Wolin \& Wolin (1993) refieren que las dimensiones de la resiliencia son:

Factor I: Denominado COMPETENCIA PERSONAL; integrado por 17 ítems que señalan: autoconfianza, autonomía, decisión, invencibilidad, mando, talento y persistencia.

Factor II: Denominado ACEPTACIÓN DE UNO MISMO Y DE LA VIDA simbolizados por 8 ítems, y manifiestan la adaptación, balance, elasticidad y un panorama de vida firme, que corresponde con la aprobación por la vida y un sentimiento de paz a pesar de la desgracia.

Epistemológicamente, se justificó esta investigación en la teoría de la Psicología Humanista de la resiliencia y teoría de la consistencia afectivo-cognoscitiva. Esta investigación aplicó conceptos y teorías de resiliencia para la mejora de los hábitos y actitudes de los estudiantes universitarios. Aprovechando las bondades de dicha teoría llegando a determinar el objetivo general de este estudio.

La teoría bifactorial de la resiliencia de Wagnild \& Young (1993) refiere que la resiliencia es una característica positiva de la personalidad, la cual permite resistir, tolerar la presión, los obstáculos y pese a ello hacer las cosas correctas. Además, puede entenderse como la capacidad de una persona, que a pesar de las condiciones de vida adversas y a las frustraciones que atraviesa, puede superarlas y salir de ellas fortalecido e incluso transformado.

Este estudio se justifica técnicamente, porque se realizó considerando que el enfoque de la universidad de estudio cuenta con un currículo por competencias, y que debería darse una mayor prioridad a la formación actitudinal, lo cual no está ocurriendo en las experiencias curriculares de muchas carreras.

Asimismo, esta investigación se justifica metodológicamente, porque se aplicó un programa de formación en resiliencia y servirá para otros docentes a fin de que puedan utilizarlo y mejorar los hábitos y actitudes en los estudiantes universitarios. De igual modo se validó el instrumento de hábitos y actitudes en los estudiantes universitarios el cual servirá para la recopilación de información en la población de Piura.

A nivel teórico esta investigación y propuesta permite ampliar los conocimientos con fundamento científico en referencia a la resiliencia en hábitos y actitudes hacia el estudio. Se considera como referencia los postulados teóricos que existen y se contrastan con la realidad particular de una determinada población del ámbito universitario.

Finalmente, el producto de la aplicación y evaluación en la institución universitaria servirá para profundizar el análisis de los resultados y así implementar estrategias que abordarán la posibilidad de iniciar nuevas investigaciones. Es importante considerar que es necesario fomentar la elaboración de programas preventivos en referencia a las dimensiones de la resiliencia ya que esto generará un impacto positivo en los hábitos y actitudes hacia el estudio, desencadenando en la mejora sustantiva en el accionar de los estudiantes y en su rendimiento académico. 


\section{METODOLOGÍA}

El diseño de investigación que se utilizó fue cuasi-experimental ya que utilizó un grupo experimental y un grupo control ya establecidos. Este diseño ofrece una ventaja porque permite comparar tanto al grupo experimental como al grupo control permitiendo así establecer si los cambios que tuvo el grupo experimental se deben al programa aplicado o a variables intervinientes (Hernández, Fernández \& Baptista, 2014).

Respecto a la población estuvo constituida por 290 estudiantes universitarios de una universidad particular de la ciudad de Piura. Así también la muestra está conformada por 70 estudiantes universitarios: 35 estudiantes para el grupo experimental y 35 estudiantes para el grupo control con características similares. Se seleccionó dos secciones que tengan características homogéneas para considerarlas en la investigación. Para ello se tomó en consideración los resultados del primer promedio obtenido por la sección en el semestre 2018-1. El muestreo es no probabilístico y por conveniencia. La técnica que se utilizó fue la encuesta, que es un documento que recoge en forma organizada los indicadores de las variables implicadas en el objetivo de la encuesta. (Casas, Repullo \& Donado, 2002). El instrumento elegido para evaluar los hábitos de estudio fue el cuestionario denominado ENCUESTA DE HÁBITOS Y ACTITUDES HACIA EL ESTUDIO (EHAHE) cuyos autores son Brown \& Holtzman (1975) y adaptado a la población mexicana por Fernando García Cortes y Eduardo García Hassey. Fue elaborado como instrumento de selección, para ayudar a la enseñanza y como instrumento de investigación. Está dividido en siete subescalas, consta de 100 ítems, 50 para medir las actitudes y 50 para medir los hábitos hacia el estudio.

\section{Análisis de datos}

En un primer paso se realizó la depuración de datos, separando los test que no fueron llenados correctamente, apartando los test que tienen doble marca, que no se llegaron a completar en su totalidad. Como segundo paso se procedió a la codificación o seriación de los cuestionarios. El tercer paso se elaboró la base de datos en Excel y luego procedió a exportar los datos al SPSS. Posteriormente se evaluó la distribución de los datos con la prueba estadística de Kolmogorov y Smirnov. Finalmente, se analizó los datos a través de tablas según la secuencialidad de los objetivos. 


\section{RESULTADOS Y DISCUSIÓN}

Tabla 1

Resultados del pretest del nivel de hábitos y actitudes hacia el estudio

\begin{tabular}{|c|c|c|c|c|c|c|c|c|c|c|c|c|}
\hline \multirow{3}{*}{ NIVEL } & \multicolumn{6}{|c|}{ Grupo Experimental } & \multicolumn{6}{|c|}{ Grupo Control } \\
\hline & \multicolumn{2}{|c|}{ GENERAL } & \multicolumn{2}{|c|}{$\begin{array}{l}\text { HABITOS } \\
\text { DE ESTU- } \\
\text { DIO }\end{array}$} & \multicolumn{2}{|c|}{$\begin{array}{c}\text { ACTITUDES } \\
\text { HACIA EL ES- } \\
\text { TUDIO }\end{array}$} & \multicolumn{2}{|c|}{ GENERAL } & \multicolumn{2}{|c|}{$\begin{array}{l}\text { HABITOS } \\
\text { DE ESTU- } \\
\text { DIO }\end{array}$} & \multicolumn{2}{|c|}{$\begin{array}{c}\text { ACTITUDES } \\
\text { HACIA EL ES } \\
\text { TUDIO }\end{array}$} \\
\hline & $\mathrm{N}$ & $\%$ & $\mathrm{~N}$ & $\%$ & $\mathrm{~N}$ & $\%$ & $\mathrm{~N}$ & $\%$ & $\mathrm{~N}$ & $\%$ & $\mathrm{~N}$ & $\%$ \\
\hline ALTO & 10 & 28,57 & 8 & 22,86 & 7 & 20 & 9 & 25,71 & 10 & 28,57 & 7 & 20 \\
\hline MEDIO & 6 & 17,14 & 5 & 14,29 & 8 & 22,86 & 13 & 37,14 & 8 & 22,86 & 9 & 25.71 \\
\hline BAJO & 19 & 54,28 & 22 & 62,86 & 20 & 57,14 & 13 & 37,14 & 17 & 48,57 & 19 & 54.29 \\
\hline TOTAL & 35 & 100 & 35 & 100 & 35 & 100 & 35 & 100 & 35 & 100 & 35 & 100 \\
\hline
\end{tabular}

Fuente: Encuesta aplicada a 70 estudiantes de la EAP de Psicología donde 35 son del grupo experimental y 35 pertenece al grupo control.

Como se puede observar en la tabla 01 en el grupo experimental el mayor porcentaje en las tres escalas se encuentra en el nivel bajo ya que a nivel general arrojó 54,28\%, en hábitos de estudio un 62,86 y en actitudes hacia el estudio arrojó un 57,14\% respectivamente. Así también, en el grupo control el mayor porcentaje en las tres escalas se encuentra en el nivel bajo ya que a nivel general arrojó 37,14\%, en hábitos de estudio un 48,57\% y en actitudes hacia el estudio arrojó un 54,29\% respectivamente.

Tabla 2

Resultados del postest del nivel de hábitos y actitudes hacia el estudio

\begin{tabular}{|c|c|c|c|c|c|c|c|c|c|c|c|c|}
\hline \multirow{3}{*}{ NIVEL } & \multicolumn{6}{|c|}{ Grupo Experimental } & \multicolumn{6}{|c|}{ Grupo Control } \\
\hline & \multicolumn{2}{|c|}{ GENERAL } & \multicolumn{2}{|c|}{$\begin{array}{c}\text { HABITOS } \\
\text { DE ESTU- } \\
\text { DIO }\end{array}$} & \multicolumn{2}{|c|}{$\begin{array}{c}\text { ACTITUDES } \\
\text { HACIA EL } \\
\text { ESTUDIO }\end{array}$} & \multicolumn{2}{|c|}{ GENERAL } & \multicolumn{2}{|c|}{$\begin{array}{c}\text { HABITOS } \\
\text { DE ESTUDIO }\end{array}$} & \multicolumn{2}{|c|}{$\begin{array}{c}\text { ACTITUDES } \\
\text { HACIA EL } \\
\text { ESTUDIO }\end{array}$} \\
\hline & $\mathrm{N}$ & $\%$ & $\mathrm{~N}$ & $\%$ & $\mathrm{~N}$ & $\%$ & $\mathrm{~N}$ & $\%$ & $\mathrm{~N}$ & $\%$ & $\mathrm{~N}$ & $\%$ \\
\hline ALTO & 24 & 68.57 & 18 & 51.43 & 25 & 71.43 & 13 & 37,14 & 11 & 31,43 & 10 & 28,57 \\
\hline MEDIO & 8 & 22.86 & 12 & 34.29 & 6 & 17.14 & 14 & 40 & 15 & 42,86 & 13 & 37,14 \\
\hline BAJO & 3 & 8.57 & 5 & 14.28 & 4 & 11.43 & 8 & 22.86 & 9 & 25,71 & 12 & 34,29 \\
\hline TOTAL & 35 & 100 & 35 & 100 & 35 & 100 & 35 & 100 & 35 & 100 & 35 & 100 \\
\hline
\end{tabular}

Fuente: Encuesta aplicado a 70 estudiantes de Psicología donde 35 son del grupo experimental y 35 pertenece al grupo control. 
Como se puede observar en la tabla 02 en el grupo experimental el mayor porcentaje en las tres escalas se encuentra en el nivel alto ya que a nivel general arrojó 68,57\%, en hábitos de estudio un 51,43\% y en actitudes hacia el estudio arrojó un 71,43\% respectivamente. Así también, en el grupo control el mayor porcentaje en las tres escalas se encuentra en el nivel medio ya que a nivel general arrojó 40\%, en hábitos de estudio un 42,86\% y en actitudes hacia el estudio arrojó un 37,14\% respectivamente.

\section{Tabla 3}

Análisis descriptivo de datos estadísticos para determinar la influencia programa de formación resiliente en los hábitos y actitudes hacia el estudio de manera general.

\begin{tabular}{|c|c|c|c|c|}
\hline & \multicolumn{2}{|c|}{ Grupo Experimental } & \multicolumn{2}{|c|}{ Grupo Control } \\
\hline & Pre test & Postest & Pre test & Postest \\
\hline $\mathrm{n}^{\mathrm{o}}$ & 35 & 35 & 35 & 35 \\
\hline Media & 163,4571 & 150,8286 & 178,4571 & 159,2 \\
\hline Dif. de medias & \multicolumn{2}{|c|}{12,6285} & \multicolumn{2}{|c|}{19,2571} \\
\hline Desv. Tipica & 34,082 & 31,093 & 31,4973 & 32,9954 \\
\hline Varianza & 1161,5496 & 966,7933 & 1088,6941 & 992,0790 \\
\hline $\begin{array}{l}\text { Coeficiente de va- } \\
\text { riación }\end{array}$ & 0,208507 & 0,206147 & 0,176498 & 0,2072575 \\
\hline Grados de libertad & \multicolumn{2}{|c|}{34} & \multicolumn{2}{|c|}{34} \\
\hline $\begin{array}{l}\text { Estadístico t calcu- } \\
\text { lado }\end{array}$ & \multicolumn{2}{|c|}{1,6437} & \multicolumn{2}{|c|}{2,35100} \\
\hline Estadístico $t$ tabular & \multicolumn{2}{|c|}{1,6909} & \multicolumn{2}{|c|}{1,6909} \\
\hline $\mathrm{p}$ & \multicolumn{2}{|c|}{0,05} & \multicolumn{2}{|c|}{0,05} \\
\hline $\begin{array}{l}\text { Estadístico t calcu- } \\
\text { lado }\end{array}$ & \multicolumn{4}{|c|}{2,498} \\
\hline Sig. (bilateral) & \multicolumn{4}{|c|}{0,015} \\
\hline $\mathrm{p}$ & \multicolumn{4}{|c|}{0,05} \\
\hline
\end{tabular}

Según los resultados de la tabla 3, se puede referenciar que el coeficiente de variación en el grupo experimental; en el postest sus datos son más homogéneos $(0,206147)$ que en el pre test $(0,208507)$. A diferencia en el grupo control, donde el postest sus datos son más heterogéneos $(0,2072575)$ que en el pre test $(0,176498)$. Así también en el grupo experimental la $t$ calculada es menor que la t tabulada (tc: $1,6437<$ tc: 1,6909$)$ lo que nos indica que el programa de formación resiliente influye en los estudiantes de una universidad particular de Piura en los hábitos y actitudes hacia el estudio, a diferencia del grupo control la t calculada es mayor que la t tabulada (tc: $2,35100>$ tc: 1,6909 ) lo que nos indica que no hay influencia. De igual forma al establecer la $t$ de muestras independientes los resultados arrojaron una Sig. (bilateral) de 0,015 menor al valor p de 0,05 lo que significa que si hay influencia. 


\section{Tabla 4}

Análisis descriptivo de datos estadísticos para determinar la influencia del programa de formación en resiliencia en los hábitos de estudio.

\begin{tabular}{|c|c|c|c|c|}
\hline & \multicolumn{2}{|c|}{ Grupo Experimental } & \multicolumn{2}{|c|}{ Grupo Control } \\
\hline & Pre test & Postest & Pre test & Postest \\
\hline$n^{o}$ & 35 & 35 & 35 & 35 \\
\hline Media & 82,9143 & 85,0571 & 76,5714 & 78,0571 \\
\hline Dif. de medias & \multicolumn{2}{|c|}{2,1428} & \multicolumn{2}{|c|}{1.4857} \\
\hline Desv. Tipica & 18,0837 & 14,3935 & 18,5889 & 13,5624 \\
\hline Varianza & 327,0218 & 207,1731 & 345,5462 & 183,9378 \\
\hline $\begin{array}{l}\text { Coeficiente de va- } \\
\text { riación }\end{array}$ & 0,218101 & 0,169221 & 0,24276 & 0,17374 \\
\hline Grados de libertad & \multicolumn{2}{|c|}{34} & \multicolumn{2}{|c|}{34} \\
\hline $\begin{array}{l}\text { Estadístico t cal- } \\
\text { culado }\end{array}$ & \multicolumn{2}{|c|}{0,5106} & \multicolumn{2}{|c|}{2,4019} \\
\hline $\begin{array}{l}\text { Estadístico t ta- } \\
\text { bular }\end{array}$ & \multicolumn{2}{|c|}{1,6909} & \multicolumn{2}{|c|}{1,6909} \\
\hline $\mathrm{p}$ & \multicolumn{2}{|c|}{0,05} & \multicolumn{2}{|c|}{0,05} \\
\hline $\begin{array}{l}\text { Estadístico t cal- } \\
\text { culado }\end{array}$ & \multicolumn{4}{|c|}{1,331} \\
\hline Sig. (bilateral) & \multicolumn{4}{|c|}{0,018} \\
\hline $\mathrm{p}$ & \multicolumn{4}{|c|}{0,05} \\
\hline
\end{tabular}

Según los resultados de la tabla 4 nos hace referencia en hábitos de estudio que el coeficiente de variación en el grupo experimental; en el postest sus datos son más homogéneos $(0,169221)$ que en el pre test $(0,218101)$. A diferencia en el grupo control, donde el postest sus datos son más heterogéneos $(0,24276)$ que en el pre test $(0,17374)$. Así también en el grupo experimental la $t$ calculada es menor que la t tabulada (tc: $0,5106<\mathrm{tt}: 1,6909)$ lo que nos indica que el programa de formación resiliente influye en los estudiantes de una universidad particular de Piura en los hábitos hacia el estudio, a diferencia del grupo control la $t$ calculada es mayor que la t tabulada (tc: $2,4019>\mathrm{tt}: 1,6909$ ) lo que nos indica que no hay influencia. De igual forma al establecer la $t$ de muestras independientes los resultados arrojaron una Sig. (bilateral) de 0,018 menor al valor p de 0,05 lo que significa que si hay influencia. 


\section{Tabla 5}

Análisis descriptivos de datos estadísticos para determinar la influencia programa de formación en resiliencia en las actitudes hacia el estudio

\begin{tabular}{|c|c|c|c|c|}
\hline & \multicolumn{2}{|c|}{ Grupo Experimental } & \multicolumn{2}{|c|}{ Grupo Control } \\
\hline & Pre test & Postest & Pre test & Postest \\
\hline $\mathrm{n}^{\mathrm{o}}$ & 35 & 35 & 35 & 35 \\
\hline Media & 80,5429 & 93,4 & 74,2571 & 78,4286 \\
\hline Dif. de medias & \multicolumn{2}{|c|}{12,8571} & \multicolumn{2}{|c|}{4.1715} \\
\hline Desv. Tipica & 17,5723 & 19,6891 & 16,0722 & 17,2410 \\
\hline Varianza & 308,7849 & 387,659 & 258,3143 & 297,2521 \\
\hline $\begin{array}{l}\text { Coeficiente de va- } \\
\text { riación }\end{array}$ & 0,21817 & 0,21081 & 0,21643 & 0,21983 \\
\hline Grados de libertad & \multicolumn{2}{|c|}{34} & \multicolumn{2}{|c|}{34} \\
\hline $\begin{array}{l}\text { Estadístico t cal- } \\
\text { culado }\end{array}$ & \multicolumn{2}{|c|}{0,819} & \multicolumn{2}{|c|}{2,0240} \\
\hline $\begin{array}{l}\text { Estadístico t ta- } \\
\text { bular }\end{array}$ & \multicolumn{2}{|c|}{1,6909} & \multicolumn{2}{|c|}{1,6909} \\
\hline $\mathrm{p}$ & \multicolumn{2}{|c|}{0,05} & \multicolumn{2}{|c|}{0,05} \\
\hline $\begin{array}{l}\text { Estadístico t cal- } \\
\text { culado }\end{array}$ & \multicolumn{4}{|c|}{3,204} \\
\hline Sig. (bilateral) & \multicolumn{4}{|c|}{0,002} \\
\hline $\mathrm{p}$ & \multicolumn{4}{|c|}{0,05} \\
\hline
\end{tabular}

Según los resultados de la tabla 5 nos hace referencia a que el coeficiente de variación en el grupo experimental; en actitudes hacia el estudio del postest sus datos son más homogéneos $(0,21081)$ que en el pre test $(0,21817)$. A diferencia en el grupo control, donde en el postest sus datos son más heterogéneos $(0,21983)$ que en el pre test $(0,21643)$. Así también en el grupo experimental la t calculada es menor que la t tabulada (tc: $0,819<$ tc: 1,6909$)$ lo que nos indica que el programa de formación resiliente influye en los estudiantes de una universidad particular de Piura en las actitudes hacia el estudio, a diferencia del grupo control la t calculada es mayor que la t tabulada (tc: $2,0240>$ tc: 1,6909$)$ lo que nos indica que no hay influencia. De igual forma al establecer la $t$ de muestras independientes los resultados arrojaron una Sig. (bilateral) de 0,002 menor al valor p de 0,05 lo que significa que si hay influencia. 


\section{CONCLUSIONES}

El programa de formación resiliente influye significativamente en los hábitos y actitudes hacia el estudio en los estudiantes de una universidad particular de Piura.

El nivel de hábitos y actitudes hacia el estudio antes de aplicar el programa de formación resiliente fue bajo a un $54,28 \%$ en la orientación al estudio, $62,86 \%$ en los hábitos para el estudio y un $57,14 \%$ en actitudes hacia el estudio respectivamente. Estos resultados reflejaron que los evaluados tuvieron una deficiencia a nivel de orientación al estudio, hábitos y actitudes hacia el estudio.

El nivel de hábitos y actitudes hacia el estudio después de aplicar el programa de formación resiliente es alto a un $68,57 \%$ en la orientación hacia el estudio, $51,43 \%$ en hábitos de estudio un y $71,43 \%$ en actitudes hacia el estudio respectivamente. Esto refleja que los evaluados tienen un mejor nivel tanto en su orientación al estudio, hábitos y actitudes hacia el estudio a diferencia que el grupo control que solo llegó a alcanzar un nivel medio.

Según el análisis descriptivo de datos estadísticos se determinó que el programa de formación resiliente influye en los hábitos y actitudes hacia el estudio en los estudiantes universitarios de Piura.

Según el análisis descriptivo de datos estadísticos se determinó la influencia programa de formación resiliente en los hábitos hacia el estudio en los estudiantes universitarios de Piura.

Según el análisis descriptivo de datos estadísticos se determinó la influencia programa de formación resiliente en las actitudes hacia el estudio en los estudiantes universitarios de Piura.

La investigación realizada en Piura para determinar la influencia del Programa de formación resiliente en hábitos y actitudes hacia el estudio ha demostrado que puede mejorar el desenvolvimiento afectivo de estudiantes durante su paso por la universidad. Los talleres aplicados en 12 sesiones influyen positivamente es esa mejora.

La propuesta innovadora de este trabajo consiste en plantear el establecimiento, dentro del plan de estudios, en los primeros semestres de las universidades, un Programa de formación resiliente para mejorar hábitos y actitudes hacia el estudio en los estudiantes, logrando que de esta manera reforzar la ecuanimidad, la perseverancia, la confianza en sí mismo, la satisfacción personal y el sentirse bien solo.

\section{RECOMENDACIONES}

Se recomienda que este programa sea aplicado con aquellos estudiantes que tengan un rendimiento académico deficiente debido a problemas de adaptación universitaria. Que no sea aplicado a estudiantes con algún trastorno de personalidad o de conducta ya que estas personas requieren de una intervención más especializada y multidisciplinaria. Que su uso sea en las horas de tutoría ya que la programación se presta para ser aplicado siguiendo normativas actitudinales. Y, que las sesiones sean aplicadas secuencialmente ya que su interrupción podría afectar a los resultados. 


\section{REFERENCIAS}

Brown, F. (1973). Curso para el estudio efectivo.Trillas.

Brown, F., \& Holtzman, W. (1975). Encuesta de hábitos y actitudes hacia el estudio (manual). Trillas.

Casas, J; Repullo, J, \& Donado, J. (2002). La encuesta como técnica de investigación. Elaboración de cuestionarios y tratamiento estadístico de los datos. Trabajo realizado con una Beca Modalidad Formación, Nivel Perfeccionamiento del Instituto de Salud Carlos III, España.

Corella, M. I. (1996). Aplicación de un Modelo de trayectoria para explicar el rendimiento académico. Revista Educación, 20(2), 41-50. https://revistas.ucr.ac.cr/index.php/ educacion/article/download/7983/7610

Escobar, M. (1996). Hábitos de Estudio en alumnos de $5^{\circ}$ semestre de Psicología de la UNAM [tesis de licenciatura, Universidad Nacional Autónoma de México].

García, A., \& Rodríguez, S. (2013). Cambio actitudinal de estudiantes de octavo grado hacia el aprendizaje de interacciones biológicas mediante la resolución de problemas [ponencia en congreso internacional]. Universidad Distrital Francisco José de Caldas, Colombia. https://ddd.uab.cat/pub/edlc/edlc_a2013nExtra/edlc_ a2013nExtrap1478.pdf

Grotberg, E. (1995). A guide to promothing resilience in children. Fundación Bernard Van Leer.

Hernández, R., Fernández, C, \& Baptista, P. (2014). Metodología de la investigación. Mc Graw Hill.

Vanistendael, S.; \& Lecomte, J. (2002). La felicidad es posible. Despertar en niños maltratados la confianza en sí mismos: construir la resiliencia. Gedisa.

Wagnild, G., \& Young, H. (1993) Escala de Resiliencia. Estados Unidos.

Wolin, S. J., \& Wolin, S. (1993): The resilient self: how survivors of troubled families rise above adversity. Villard Books. 